\title{
Spatial '-omics' in large samples using high numerical aperture oblique plane microscopy
}

Peter Brown, Rory Kruithoff, Lei Zhou, Douglas Shepherd Arizona State University, USA

\section{Abstract Text}

There are multiple large scale efforts, including the Human Cell Atlas and the Allen Brain Atlas, attempting to create spatial atlases of cell identity and location in entire organs. New highly-multiplexed molecular mapping methods that perform '-omics' using various readout strategies are continually evolving to achieve this lofty goal. Those strategies that rely on fluorescent microscopy require multiple rounds of repeated imaging of the same sample volume due to the spectral overlap of available fluorophores. Between sequential imaging rounds, an automated fluidics system performs chemical exchange of fluorescent labels between molecular targets. Postacquisition, the obtained data are computationally decoded to determine the unique identity of individual molecules.

The requirements for sequential multiplexed molecular imaging using fluorescent microscopy are high numerical aperture (NA), large area 3D sample scanning for thin samples, multiple laser lines, a sealed sample chamber with fluidic input/output, and a fluidics controller. This unique set of requirements has led to a reliance on traditional fluorescent imaging methods, such as confocal or epifluorescence microscopy. To speed the imaging time for traditional methods, many groups intentionally under-sample in both the lateral and axial dimensions. Even with under-sampling, the imaging time dominates the total experimental time for larger samples.

Light sheet fluorescence microscopy is a natural choice to increase speed, sampling, and optical sectioning. However, sequential multiplexed molecular imaging's unique requirements rule out the existing single objective, oblique plane, open-top, and high NA dual objective light sheet approaches. A new light sheet approach that 
meets the stated requirements would transform the accuracy, speed, and application of fluorescent microscopy for sequential multiplexed molecular imaging. High-quality data generation would also enable advancements in computational decoding approaches, analogous to the computational advancements currently taking place in singlemolecule localization microscopy.

Building on recent advancements in oblique plane microscopy (OPM [1]), we created a high NA OPM with an integrated fluidics unit for sequential multiplexed molecular imaging. Our approach translates the sample mounted in a fluidics chamber through a static light sheet and uses a solid immersion objective as the tertiary imaging objective [2]. We quantitatively evaluated our high NA OPM design's volume performance using calibration samples and a simplified forward model. Turning to real samples, we quantified the coexpression of RNA for the SARS-CoV-2 entry genes ACE2 and TMPRSS2 in alveolar type II cells across centimeter-scale fixed human lung tissue slices. As constructed, our high NA OPM can image $\sim 1 \mathrm{~cm} \times 1 \mathrm{~cm} \times 50 \mu \mathrm{m} \times 1$ color per hour at single-molecule resolution. The fluidic chamber optical window size places a practical constraint on the otherwise unconstrained lateral imaging size. We anticipate this increase in resolution, sampling, and speed versus traditional fluorescent microscopy methods will benefit spatial molecular and structural atlas efforts. 